\title{
FACIAL EXPRESSION RECOGNITION BASED ON WAPA AND OEPA FASTICA
}

\author{
Humayra Binte $\mathrm{Ali}^{1}$ and David M W Powers ${ }^{2}$ \\ ${ }^{1}$ Computer Science, Engineering and Mathematics School, Flinders University, Australia \\ ${ }^{2}$ Computer Science, Engineering and Mathematics School, Flinders University, Australia
}

\begin{abstract}
Face is one of the most important biometric traits for its uniqueness and robustness. For this reason researchers from many diversified fields, like: security, psychology, image processing, and computer vision, started to do research on face detection as well as facial expression recognition. Subspace learning methods work very good for recognizing same facial features. Among subspace learning techniques PCA, ICA, NMF are the most prominent topics. In this work, our main focus is on Independent Component Analysis (ICA). Among several architectures of ICA, we used here FastICA and LS-ICA algorithm. We applied Fast-ICA on whole faces and on different facial parts to analyze the influence of different parts for basic facial expressions. Our extended algorithm WAPA-FastICA and OEPA-FastICA are discussed in proposed algorithm section. Locally Salient ICA is implemented on whole face by using $8 \times 8$ windows to find the more prominent facial features for facial expression. The experiment shows our proposed OEPAFastICA and WAPA-FastICA outperform the existing prevalent Whole-FastICA and LS-ICA methods.
\end{abstract}

\section{KEYWORDS}

ICA-Independent Component Analysis, FER-Facial Expression Recognition, OEPA-Optimal Expression specific Parts Accumulation. WAPA-Weighted All Parts Accumulation algorithm.

\section{INTRODUCTION}

This work presents a detailed examination of the use of Independent Component Analysis (ICA) for feature extraction of facial expression recognition. Mathematical and analytical investigation of ICA is given here in detail. Experimental verification of the behavior of ICA with Euclidean distance classifiers is performed here. There are several architectures to implement ICA.

In this research work, we implemented the FastICA method on facial image dataset with different kernels, like hyper tangent, Gaussian and cubic kernel. We pre-processed the data using most basic centering method. Then we applied it on whole face and facial parts. For fusion we used Optimal Expression-specific Parts Accumulation (OEPA) and Weighted All Parts Accumulation (WAPA) technique that we described later. The OEPA-FastICA outperforms all other methods. We also implemented LS-ICA on the same images and successfully made comparisons with FastICA, WAPA-FastICA and OEPA-FastICA. For LS-ICA 8x8 window is applied for scanning process and finding ICs. 
International Journal of Artificial Intelligence \& Applications (IJAIA), Vol. 5, No. 3, May 2014

\section{RELATED WORK}

The work from [10] claims that the structural information of sensory inputs stays in the redundancy if the sensory input system. PCA and ICA are the most well known methods for redundancy as well as finding useful components for attaining distinguishable properties. This redundancy provides information to develop a factorial system and independent components (ICs) develop from this representation. Complex object of higher order dependencies need such representation to be encoded. Independent component representation from this sort of redundant data is a general coding strategy for the visual systems [12].

The most prominent subspace learning algorithms are PCA, ICA, NMF, LDA etc. For feature extraction from the facial expression images, most of the early FER research works extracted useful features using Principal Component Analysis (PCA). PCA is a second-order statistical method, which creates the orthogonal bases. These orthogonal bases provide the maximum variability. These variables are good source fro distinguishing features in image analysis. It is also commonly used for dimension reduction. [13] and [14] employed PCA as one of the feature extractors to solve FER with the Facial Action Coding System (FACS). We previously investigated PCA on facial expression recognition [26] and [27]. The work in [26] and [27] shows applying PCA on face parts rather whole face gives more accuracy to recognize expressions from facial image data.

In this work, we investigate ICA on facial data to recognize basic facial expressions. Independent Component Analysis (ICA) has the ability to extract local facial features [5], [15] . In recent years ICA has been extensively utilized for FER [5], [15], [16]. As much of the information that distinguishes different facial expressions stays in the higher order statistics of the images [16], ICA is a better choice for FER than PCA. In [19], Bartlett et al. implemented ICA to recognize twelve different facial expressions referred to FACS. In [20], Chao-Fa and Shin utilized ICA to extract the IC features of facial expression images to recognize the Action Units (AU) in the whole face as well as the lower and upper part of the faces. However, the FAU-based works mostly focus on the successful extraction of FAUs rather than the recognition of emotions derived from facial expression changes. Also, they encounter the limitation of AUs due to the fact that the separate facial expressions do not directly draw the comparisons with human data [21]. In [15], Buciu et al. proposed ICA for the emotion-specified facial expression recognition and applied ICA on the Japanese female facial expression database [22]. In [23], Bartlett et al. again introduced ICA on the PCs for face recognition in two different architectures where the first architecture finds the spatially local basis images and the second one the factorial face codes. They showed that both the architectures outperform PCA. Applying ICA on the PC features is usually recognized as Enhanced Independent Component Analysis (EICA) [24]. In the experiment of [24], Liu used frontal face of more than thousand images from FERET dataset and applied EICA on those dataset. They found EICA efficient for FER process. Basically EICA implements ICA on the principal component decomposed features and its efficient for content based image retrieval. 
International Journal of Artificial Intelligence \& Applications (IJAIA), Vol. 5, No. 3, May 2014

\section{INDEPENDENT COMPONENT ANALYSIS}

The principal component analysis (PCA) performs by the Karhunen-Loeve transformation and produces mutually uncorrelated features y (i), $i=0,1 \ldots \mathrm{N}$. When the goal of image or data processing is to reduce the dimension as well as to minimize the mean-square error, KarhunenLoeve transformation is a good solution. However, certain applications, such as the one depicted in Figure 1, the mathematical or analytical solution does not meet the expectations. In addition the more recently development of Independent Component Analysis (ICA) algorithm seeks to achieve much more complicated features than simple decorrelation of data or image analysis [Hvya 01, Como 94, Jutt 91, Hayk 00, Lee 98]. Then ICA task is defined as follows: Given the set of input samples $\mathrm{x}$, determine an $\mathrm{M} \times \mathrm{M}$ invertible matrix $\mathrm{W}$ such that the entries $\mathrm{y}(\mathrm{i}), \mathrm{i}=0$, $1 . . \mathrm{M}-1$, of the transformed vector

$y=W x$

are mutually independent. The uncorrelated ness required by PCA is less important feature than the statistical independence which independence can be implemented by ICA algorithms. Only for Gaussian random variables, this two conditions are equivalent to each other.

Seeking for statistical independence of data gives the mean of exploiting a lot more information, which lies in the higher order statistics of the data. As the example of Figure 1 shows, trying to search by analyzing information in the second order statistics only results in the least interesting, projection direction, that is, that of a0. However, a1 is the most suitable direction from the class separation point of view. Additionally, implementing ICA can unveil the higher order statistics of the data the piece of information that point's a1 as the most suitable direction. Furthermore, the cognitive nature of human brain searches for statistically independent, by processing the (input) sensory data [7]. Barlow [8] proposes the outcome of the early processing performed in our visual cortical feature detector systems might be the result of a redundancy reduction process.

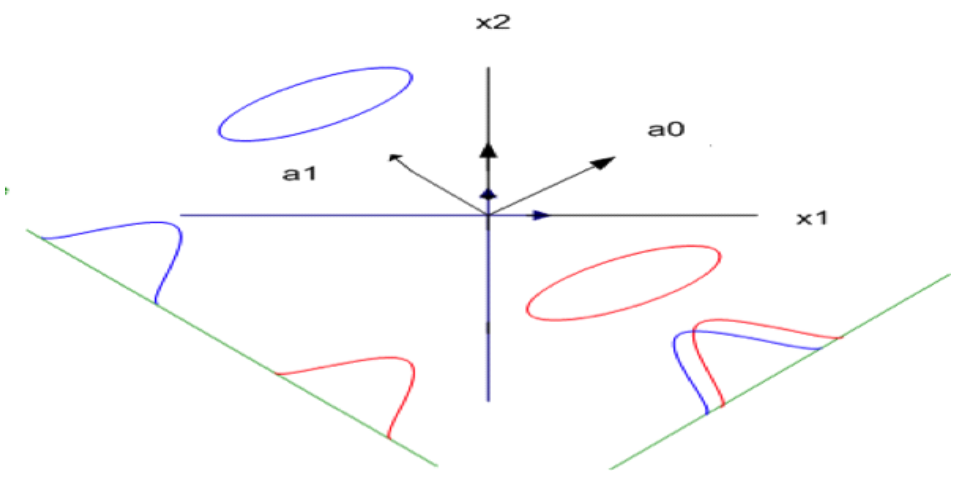

Figure 1. This example shows projection on the eigenvector with the larger eigenvalue keeps the two classes' ad joint. But projection on the other eigenvectors keeps the classes distinguishable from each other.

Before developing the techniques for performing ICA, we need be confident that such a has a solution and under specific conditions. For this we have to assume that our input random data 
vector $\mathrm{x}$ is principally generated by a linear combination of statistically independent sources, which is.

$x=A y$

Now the task is to exploit the information of $\mathrm{x}$ to define under what conditions the matrix $\mathrm{W}$ can be computed so as to recover the components of $y$ from equation of (2). Usually A is mixing matrix and $\mathrm{W}$ is the demixing matrix.

The first condition is all independent components y (i), $\mathrm{i}=1,2 \ldots \mathrm{N}$, must be non-Gaussian and the second condition is that matrix A must be invertible. It means, in contrast to PCA, ICA is meaningful only if the random variables are non-Gaussian. And mathematically for Gaussian random variables, statistical independence is equivalent to the uncorrelatedness nature of PCA. So we have to assume that the obtained Independent Components y (i), $\mathrm{i}=1,2 \ldots \mathrm{N}$, are all Gaussian, then by using any unitary matrix, a linear transformation will be a solution [7]. On the other hand, by imposing a specific orthogonal structure onto this transformation matrix, PCA achieves a unique solution.

\section{ICA AS FEATURE EXTRACTION}

In a specific task like face recognition the most important information may be hidden in higher order statistics, not just the second order statistics. An example would be phase and amplitude spectrums. Amplitude Spectrum of an image is captured by Second-order statistics and the phase spectrum is hidden in the higher order statistics. The phase spectrum includes a great deal of information and also applies the human perception.

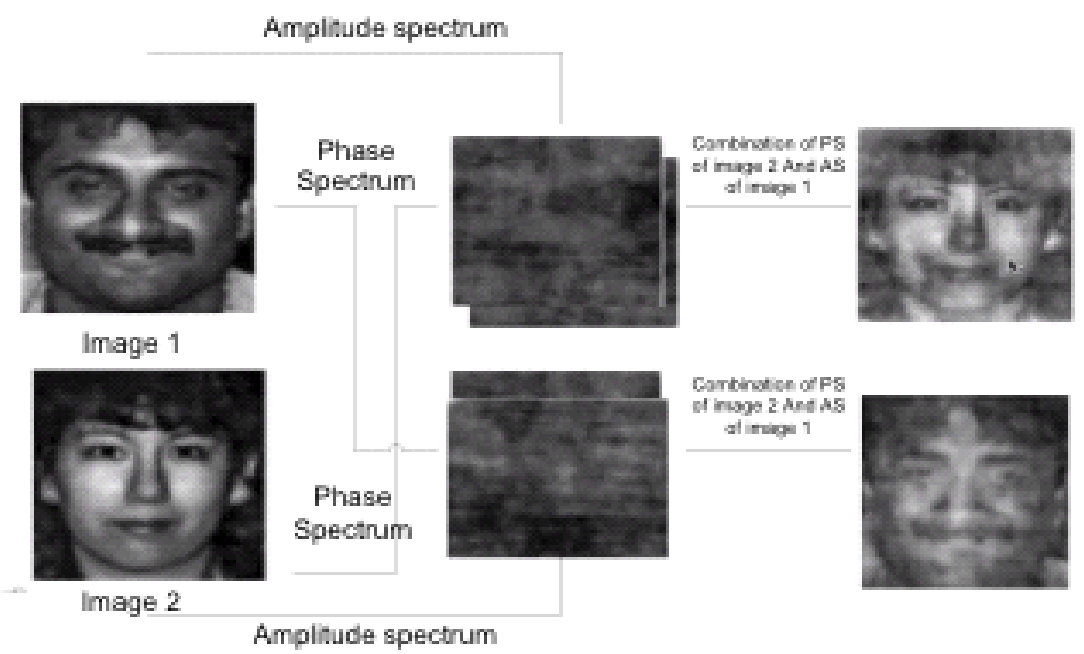

Figure 2. [PS: Phase Spectrum, AS: Amplitude Spectrum] The phase spectrum of the image 2 and Amplitude spectrum of image 1 produces the blurred image of image 2 . The phase spectrum of the image 1 and Amplitude spectrum of image 2 produces the blurred image of image 1 replicated from [9].

The above figure (Figure 2) shows that the more information pertaining the human eye is the phase spectrum. 


\section{ICA Algorithm}

\subsection{ICA By Maximization Of Non Gaussianity}

Non-Gaussian components are Independent [1]. Maximization of non-Gaussianity is the simple principles for estimating ICA model. Under certain conditions, the distribution of a sum of independent random variables have a propensity towards a Gaussian distribution, which is the concept of central limit theorem. By finding the right linear combinations of the mixture variables, independent components can be estimated. The mixing can be inverted as,

$S=A^{-1} X$

Now it becomes,

$Y=b^{T} X=b^{T} A S$

Here $\mathrm{b}$ stand for one of the rows $A^{-1}$, but this linear combination $b^{T} X$ actually stand for one of the independent components. But as we have no knowledge of matrix A, we cannot determine such 'b', although we can find an approximate estimator. NonGuassianity has two different practical implementations.

\subsubsection{Kurtosis}

Kurtosis or the fourth order cumulant is the classical measure of non-gaussianity. It is stated by

$$
\operatorname{Kurt}(y)=E\left\{y^{4}\right\}-3\left(E\left\{y^{2}\right\}\right)^{2}
$$

We should assume variable y to be standardized, so it can say

$$
\operatorname{Kurt}(y)=E\left\{y^{4}\right\}-3
$$

The normalized version of the fourth moment $E\left\{y^{4}\right\}$ is defined as Kurtosis. The fourth moment is equal to 3 For the Gaussian case implementation. and hence $\operatorname{Kurt}\{y\}=0$. So it means for the gaussian variable kurtosis is zero but it is non-zero for the nongaussian random variables.

Hence the kurtosis is simply a normalized version of the fourth moment. For the Gaussian case the fourth moment is equal to 3 and hence $\operatorname{Kurt}(Y)=0$. Thus for gaussian variable kurtosis is zero but for nongaussian random variable it is non-zero.

\subsubsection{Negentropy}

An alternative very important measure of nonguassianity is negentropy. From mathematical analysis, the measure of non-gaussianity is zero for a Gaussian variable and always non negative for a non Gaussian random variable. We can use a slightly modified version of the definition of differential entropy as negentropy. Negentropy $\mathrm{J}$ is defined as 
$J(y)=H\left(y_{\text {gauss }}\right)-H(y)$

Where $y_{\text {gauss }}$ is a Gaussian random variable of the same covariance matrix as .

\subsection{Negentropy in terms of Kurtosis}

The largest entropy among all the random variables is the Gaussian variable. The negentropy for the random variables is zero if and only if it is a Gaussian variable, otherwise it is always positve. Moreover, the negentropy has an extra property that it is invariant for invertible transformation. But the estimation of negentropy is difficult, as it requires an estimate of the probability density function. Therefore in practice using higher order moments approximates negentropy.

$$
J(y) \approx 1 / 12 E\left\{y^{3}\right\}^{2}+1 / 48 k u r t(y)^{2}
$$

Another approach is to generalize the higher order cumulant approximation in order to increase the robustness. Again the random variable y is assumed to be a standard variable. So that it uses expectations of general non-quadratic functions. To make it simple, we can take any two nonquadratic functions G1 \& G2 such that G1 is odd \& G2 is even \& we obtain the following approximation [equation 9].

$\mathrm{j} J(y) \approx K_{1}\left(E G_{1}(y)\right)^{2}+K_{2}\left(E G_{2}(y)\right)-E G_{2}(U)^{2}$

Where $\mathrm{K} 1 \& \mathrm{~K} 2$ are positive constant \& $\mathrm{U}$ is standardized Gaussian variable.

\section{FAST FIXED POINT Algorithm FOR ICA (FASTICA)}

Assume that we have a collection of prewhitened random vector $x$. Using the derivation of the preceding section, the following steps show the fast fixed point algorithm for ICA.

1. Take a random initial vector $\mathrm{w}(0)$ of norm 1.Let $k=1$.

2. Let $w(k)=E\left\{x\left(w(k-1)^{T} x\right)^{3}\right\}-3 w(k-1)$. Byusing a large sample of $x$ vectors, the expectation can be estimated.

3. Divide $w(k)$ by its norm.

4. If $\left|w(k)^{T} w(k-1)\right|$ is not close enough to 1 , let $k=k+1$ and go back to step 2 .

Otherwise, output the vector $w(k)$.

The final vector $w(k)$ stands for one of columns of the (orthogonal) mixing matrix $[B]$. This means that $w(k)$ separates one of the non-Gaussian source signals. The most dominant property of this algorithm is that it requires a very small number of iterations; normally 5-10 iterations seem to be enough to obtain the maximal accuracy allowed by the sample data set. This is due to the cubic convergence property of the algorithm. 


\title{
6.1. Performance Index
}

A well-known formula for measuring the separation performance is Performance Index (PI)[1] which is defined as

$$
P I(A)=\frac{1}{m(n-1)} \sum_{i=1}^{m} \sum_{j=1}^{n}\left(\frac{[A]_{i, j}}{\max _{j}[A]_{i, j}}-1\right)
$$

Where $[A]_{i, j}$ is the $(\mathrm{i}, \mathrm{j})$ th-element of the matrix $[\mathrm{A}]$. As because, the knowledge of the mixing matrix $[\mathrm{A}]$ is required, the smaller value of PI usually mentions the better performance evaluation for experimental settings.

\subsection{Proposed Algorithm}

As pre-processing we did differentiation on whole set of images. Then we applied ICA on whole faces and on different facial parts, which hold the prominent characteristics for expression detection. In [26 \& 27], we implemented PCA on whole and part based faces. In this paper we implemented ICA incorporating (i) Weighted All Parts Accumulation (WAPA) algorithm and (ii) Optimal Expression-specific Parts Accumulation (OEPA). We consider four parts left eye, right eye, mouth and nose and we found better results.

First Approach: In first option, we consider all the four parts to train an expression and utilize their weighted value in order to identify the expression from the test data.

\section{Weighted All Parts Accumulation (WAPA) algorithm Step: Apply the relevant equation to identify an expression}

\author{
--Ehap = W1.LEhap + W2.REhap + W3.Nhap + W4.Mhap \\ --Eang = W1.LEang + W2.REang + W3.Nang + W4.Mang \\ --Edis = W1.LEdis + W2.REdis + W3.Ndis + W4.Mdis \\ --Efear $=$ W1.LEfear + W2.REfear + W3.Nfear + W4.Mfear \\ -- Esur $=$ W1.LEsur + W2.REsur + W3.Nsur + W4.Msur \\ $--E_{s a d}=\mathrm{W} 1 . \mathrm{LEsad}+\mathrm{W} 2 \cdot \mathrm{REsad}+\mathrm{W} 3 . \mathrm{Nsad}+\mathrm{W} 4 \cdot \mathrm{Msad}$ \\ LE=Left eye, RE=Right Eye, M=Mouth, N=Nose In these equations, we calculate the weighted \\ average of the four parts of faces, eye (left, right), nose and mouth.
}

\section{Second approach:}

Sometimes a subset of all the four parts of the face is optimal in terms of processing time and accuracy for identifying an expression. In second approach, we adapt similar approach and named it as Optimal Expression- specific Parts Accumulation (OEPA). In case of identifying an expression, if more than one subset of four parts give almost equal accuracy within a threshold value, this algorithm picks the subset of minimal number of parts in order to reduce the processing time. It increases the efficiency of the program in terms of time and as well as accuracy. 
International Journal of Artificial Intelligence \& Applications (IJAIA), Vol. 5, No. 3, May 2014

\section{Algorithm of Optimal Expression-specific Parts Accumulation (OEPA)}

Initialization: First we initialize the random population.

\section{Evaluation:}

- --Step 1: Assume I = [i1, i2, i3, i4] is the vector of different segments of facial region, like: both eyes, mouth and nose.

- --Step 2: Evaluate fitness f (I (i)) representing the accuracy of detection based on a particular instance of I (i), where $\mathrm{i}=1$ to 4 .

- --Step 3: Assume E = [e1, e2, e3, e4, e5, e6] is the vector for six basic emotions, like: happy, sad, disgust, anger, fear and surprise. For each expression E (j), we need to run step 4 and step 5, where $\mathrm{j}=1$ to 6 .

- $\quad$--Step 4: Assume $\mathrm{P}=\mathrm{I}$ (i) for each region, where $\mathrm{i}=1$ to 4 and

$K 1=\max (f(P))$, accuracy value for detection of expression $E(j)$.

$\mathrm{K} 2=\max (\mathrm{f}(\mathrm{P} 1+\mathrm{P} 2))$, accuracy value for detection of region $\mathrm{P} 1$ and $\mathrm{P} 2$

for the expression $\mathrm{E}(\mathrm{j})$, where $\mathrm{P} 1 / \mathrm{P} 2$.

$\mathrm{K} 3=\max (\mathrm{f}(\mathrm{P} 1+\mathrm{P} 2+\mathrm{P} 3)=$ accuracy value for detection of $\mathrm{P} 1, \mathrm{P} 2$ and

$\mathrm{P} 3$ expression where $\mathrm{P} 1 /=\mathrm{P} 2 / \mathrm{P} 3$.

$\mathrm{K} 4=\max (\mathrm{f}(\mathrm{P} 1+\mathrm{P} 2+\mathrm{P} 3+\mathrm{P} 4)=$ accuracy value for detection of $\mathrm{P} 1, \mathrm{P} 2, \mathrm{P} 3$

and $\mathrm{P} 4$ expression where $\mathrm{P} 1 / \mathrm{P} 2 /=\mathrm{P} 3 / \mathrm{P} 4$.

- $\quad$-Step 5: Get the facial region $\sum P_{i}$,

for which $\mathrm{L}=\max (\mathrm{Ki}) \mid$ here $\mathrm{L}$ is the final accuracy value for the particular expression.

\section{LOCALly SALIENT ICA}

The Locally Salient Independent Component Analysis (LS-ICA) is an extension of ICA in terms of analyzing the region based local basis images. In terms of facial expression detection this method outperforms simple ICA due to its ability to identify local distortions more accurately. It needs two-step to compute the LS-ICA. First thing is to create component based local basis images based on ICA architecture and then order the obtained basis images in the order of class separability for achieving good recognition.

The LS-ICA method imposes additional localization constraint in the process of the kurtosis maximization. Thus it creates component based local basis images. Each iterative solution step is weighted. And it becomes sparser by only emphasizing larger pixel values. This sparsification contributes to localization. Let $V$ be a solution vector at an iteration step, we can define a weighted solution, $W$ where $W_{i}=\left|\left(V_{i}\right)\right|^{\beta} V_{i}$ and $W=W /\|W\| . \beta>1$ is a small constant. The kurtosis is maximized in terms of $W$ (weighted solution) instead of $V$ as in equation 11.

$$
\operatorname{Kurt}(w)=\left|E(W)^{4}-3\left(E(b)^{2}\right)^{2}\right|
$$

By applying equation 12, which is a update rule we can derive a solution for equation 11. 
$S^{t+1}=E\left|V_{i}\right|^{\beta} Q\left(\left|V_{i}\right|^{\beta} S^{T}(t) Q\right)^{3}$

Here (in equation 12) $S$ is a separating vector and $Q$ contains whitened image matrix. Then the resulting basis image is $W^{i}=\left|V_{i}\right|^{\beta}\left(S^{T} Q\right)_{i}$. Then according to the algorithm LS- ICA basis images are created from the ICA basis images selected in the decreasing order of the class separability P [11], where $P=\Omega_{\text {between }} / \Omega_{\text {within }}$. Thus both dimensional reduction and good recognition performance can be achieved. The output basis images contain localized facial features that are discriminant for change of facial expression recognition.

\section{DAtaset}

For experimental purpose we benchmark our results on Cohn Kanade Facial Expession dataset. e Nearly 2000 image sequences from over 200 subjects are in this dataset. All the expression dataset maintain a sequence from neutral to highest expressive grace. We took two highest graced expressive image of each subject. As we took 100 subjects, so the total image becomes 1200. 100 subject x 6 different expression x 2 of each expression. SO it becomes $100 \times 6$ × $2=1200$. There is a significant variation of age group, sex and ethnicity. As mentioned in original dataset copyright, two Panasonic WV3230 cameras, each connected to a Panasonic S-VHS AG-7500 video recorder with a Horita synchronized time-code generator, has been used to capture the facial expressions. Although they captured frontal image and also 30 degree rotated images, for this study we only use the frontal images. Subjects display six basic emotions, happiness, sad, anger, fear, disgust and surprise. The way of performing the action is instructed by an experimenter. The experimenter is trained about the facial action coding system (FACS). Each expression starts from neutral face to the highest peak of any expression. We use the two highest grace from every expression. So it reduces the total number of used images.

The following figure (Figure 3) shows a portion of the dataset of our experiment. The first row is the frontal faces from CK dataset. Second, third, fourth and fifth rows show mouth, left eye, and right eye and nose respectively.

First we sorted the very high and very low contrast of images. Then we adjust the brightness to make all images of average brightness. 


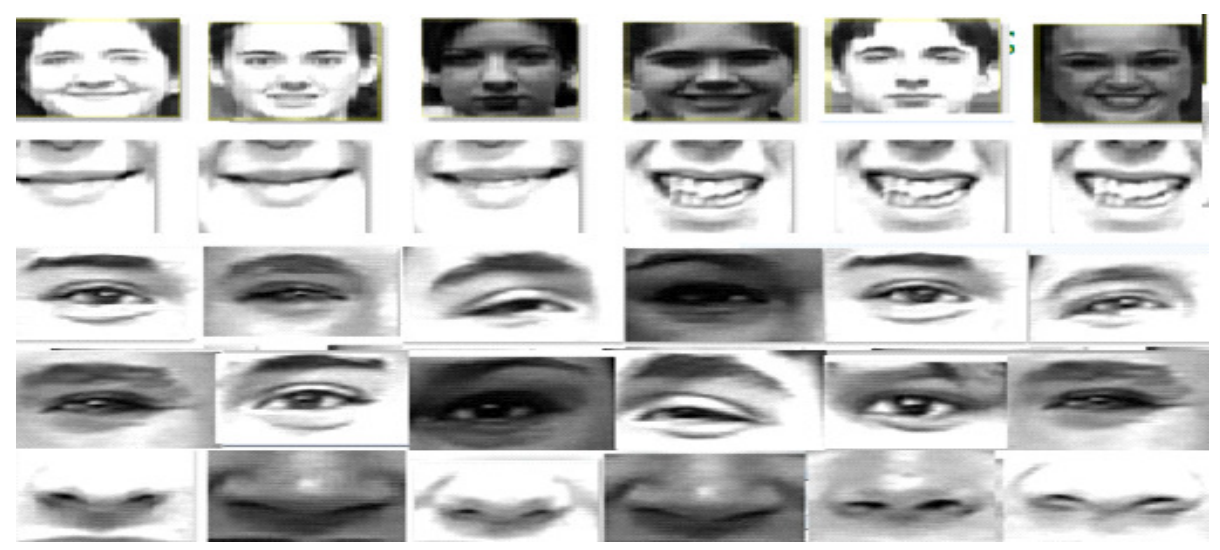

Figure 3. Original Dataset from CK database and the four parts of every image obtained from our algorithm and used as data.

\section{EXPERIMENTAL SETUP}

\subsection{Face and Facial Parts Detection}

In CK dataset, the background is large with all the face images. First we apply the Viola-Jones algorithm to find the faces. For eyes, nose and mouth detection we applied cascaded object detector with region set on already detected frontal faces [Figure 4]. This cascade object detector with proper region set can identify the eyes, nose and mouth. Actually it uses Viola-Jones Algorithm as an underlying system. This object uses a cascade of classifiers to efficiently process image regions for the presence of a target object. Each stage in the cascade applies increasingly more complex binary classifiers, which allows the algorithm to rapidly reject regions that do not contain the target. If the desired object is not found at any stage in the cascade, the detector immediately rejects the region and processing is terminated.

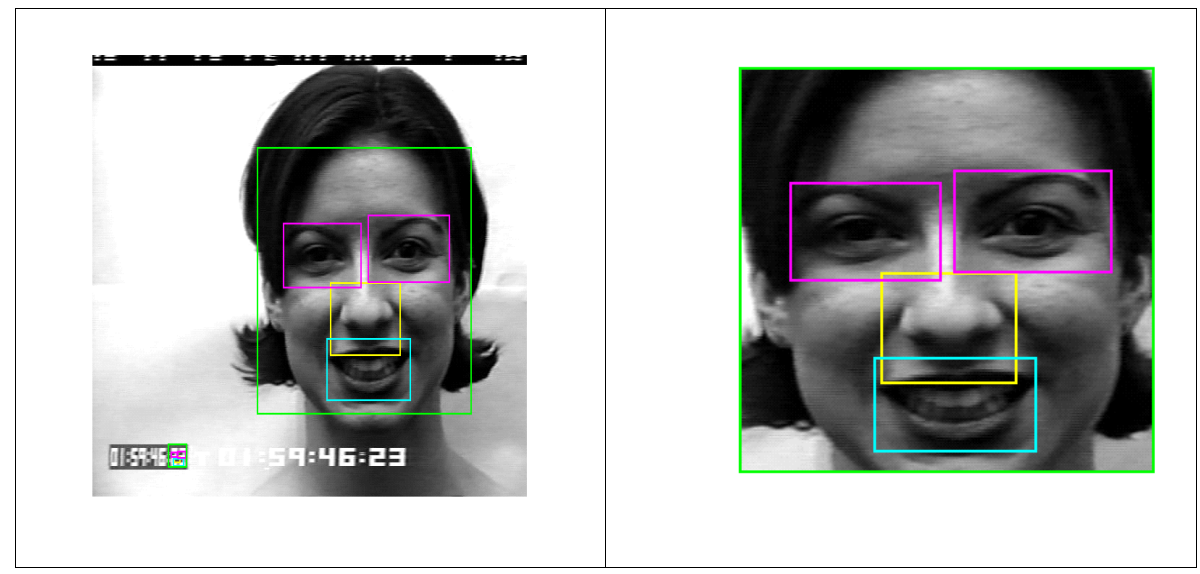

Figure 4.Finding Face and Face Parts 


\subsection{Contrast Adjustment}

The CK dataset varies greatly in image brightness. First we use Contrast Adjustment to enhance the image from very light images. Due to some reason when we apply the Histogram Equalization for very light images, the images distort. On the other side when apply Contrast Adjustment for very dark images, the images distort. As far of our knowledge, we are the first to separate the technical difference between Contrast Adjustment and Histogram Equalization. Contrast Adjustment works good for very light images as well as Histogram Equalization works good for very dark images from CK facial expression dataset.

When an image lacks sharp difference between black and white, it means the image lacks contrast. For image Contrast Adjustment, first we set an upper and lower limit of pixel value. Then we linearly scale the pixel values of facial expression images between these preset upper and lower values. But which pixel values are above or below this range, we saturated those to the upper or lower limit value, respectively. Figure 5. depicts the process of Contrast Adjustment.

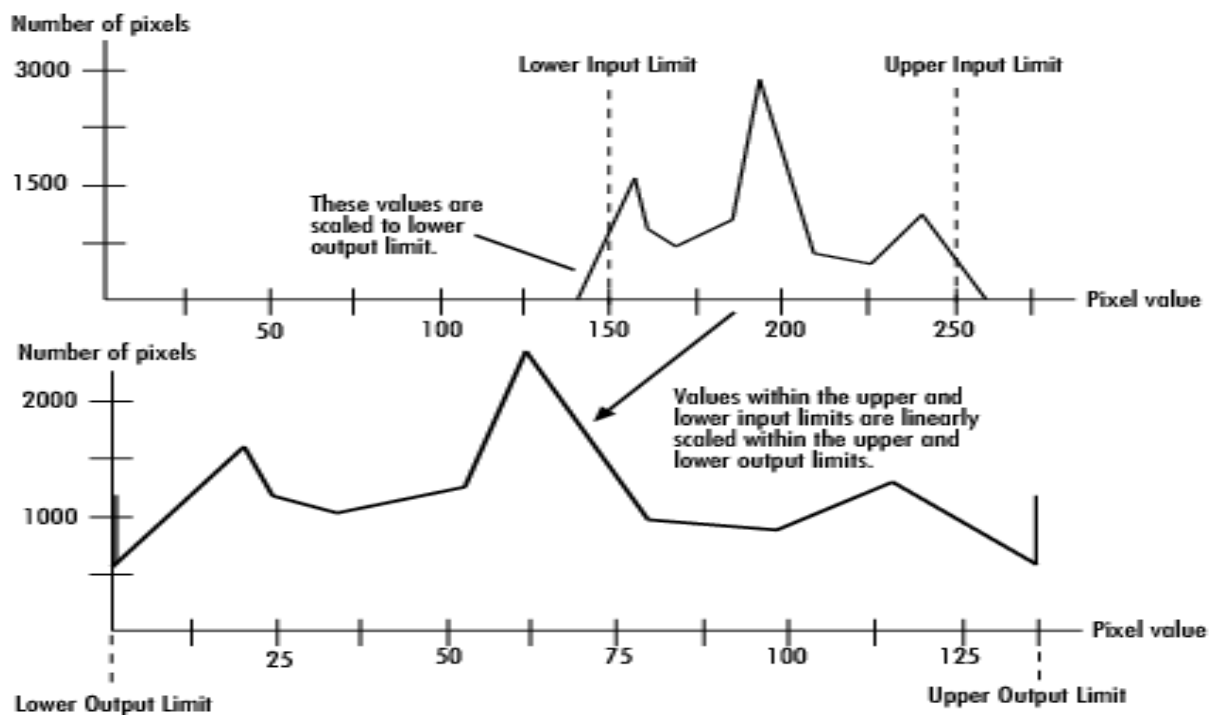

Figure 5. Contrast Adjustment Procedure

\subsection{Histogram Equalization}

As discussed in the previous subsection, to improve the contrast of the very dark images we apply Histogram equalization. We specify the histogram which is the output from the Contrast Adjustment process. By transforming the values of the dark images intensity it matches the specified histogram. The peaks of the histogram are widened and the valleys are compressed. So that the output is equalizes histogram.

\subsection{Cropping}

We set a fixed bounding box for all the faces. The face is cropped based on the given bounding box with Viola-Jones detector. Finally we cropped the face as figure as Figure 4. 


\subsection{Training and Testing Data}

We used here 100 subjects 1200 images and four face parts of the images. For every case (whole face, eyes, nose and mouth) we used $60 \%$ of the images for training and $40 \%$ for testing. We make separate face spaces for six different facial expressions. Then after ICA decomposition on the test images Euclidian distance is used for recognizing the closely match. When we applied ICA on whole faces, it happens that the system finds similar faces rather than similar expression when the same or similar person's face is in the both the train and test folder. For this reason part based analysis, WAPA, OEPA and also LS-ICA performs better than whole face ICA decomposition.

\section{Result AnALYSiS}

\subsection{FastICA}

We performed differentiation on the vector of images as a preprocessing step. Figure 6 . shows the filtered mixed signals after differentiation. Different source images are depicted in Figure 7. Independent components of the images are shown in Figure 8. Figure 9. Shows global 3D matrix of Performance Index. As described before Performance Index is a well known formula for measuring the separation performance which can be defined as

$$
P I(A)=\frac{1}{m(n-1)} \sum_{i=1}^{m} \sum_{j=1}^{n}\left(\frac{[A]_{i, j}}{\max _{j}[A]_{i, j}}-1\right)
$$

Where $[A]_{i, j}$ is the $(\mathrm{i}, \mathrm{j})$ th-element of the matrix $[\mathrm{A}]$. As because, the knowledge of the mixing matrix $[\mathrm{A}]$ is required, the smaller value of PI usually mentions the better performance evaluation for experimental settings. Finally Figure 10. Shows the estimated Inverse matrix.
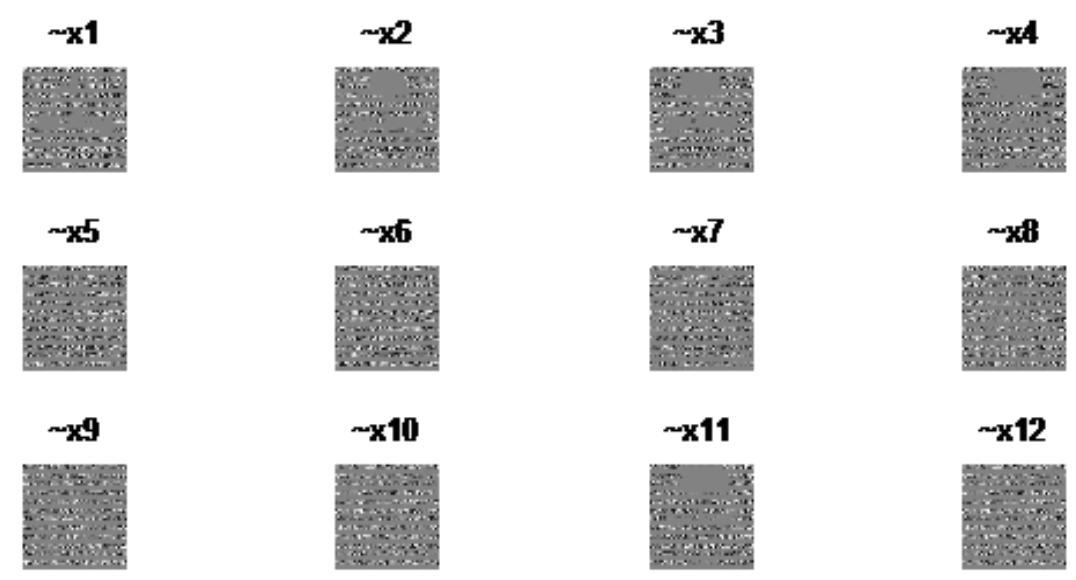

Figure 6. Filtered Mixed Signals (Differentiation) 
International Journal of Artificial Intelligence \& Applications (IJAIA), Vol. 5, No. 3, May 2014

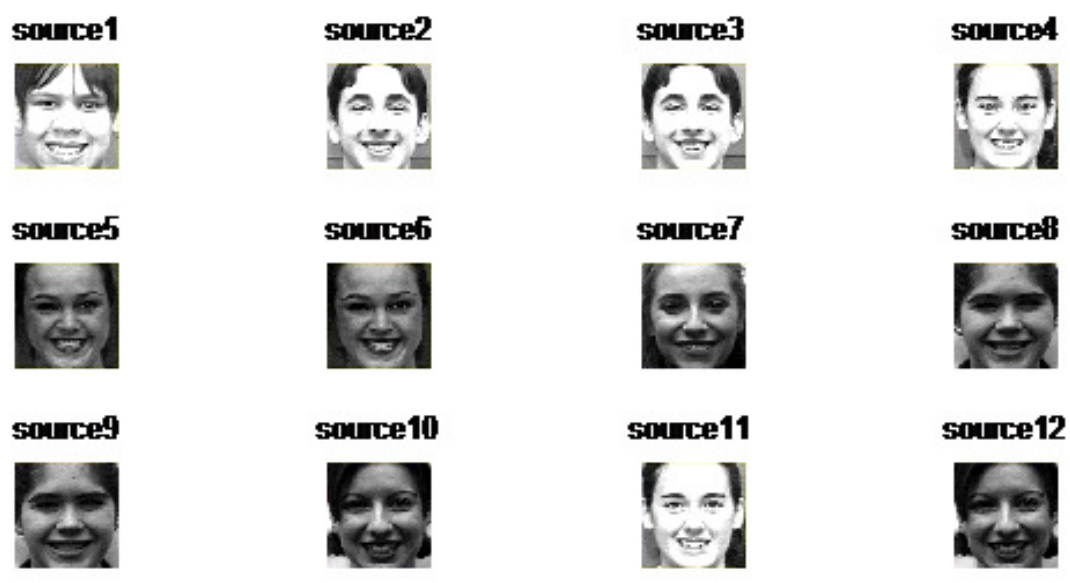

Figure 7. Source images

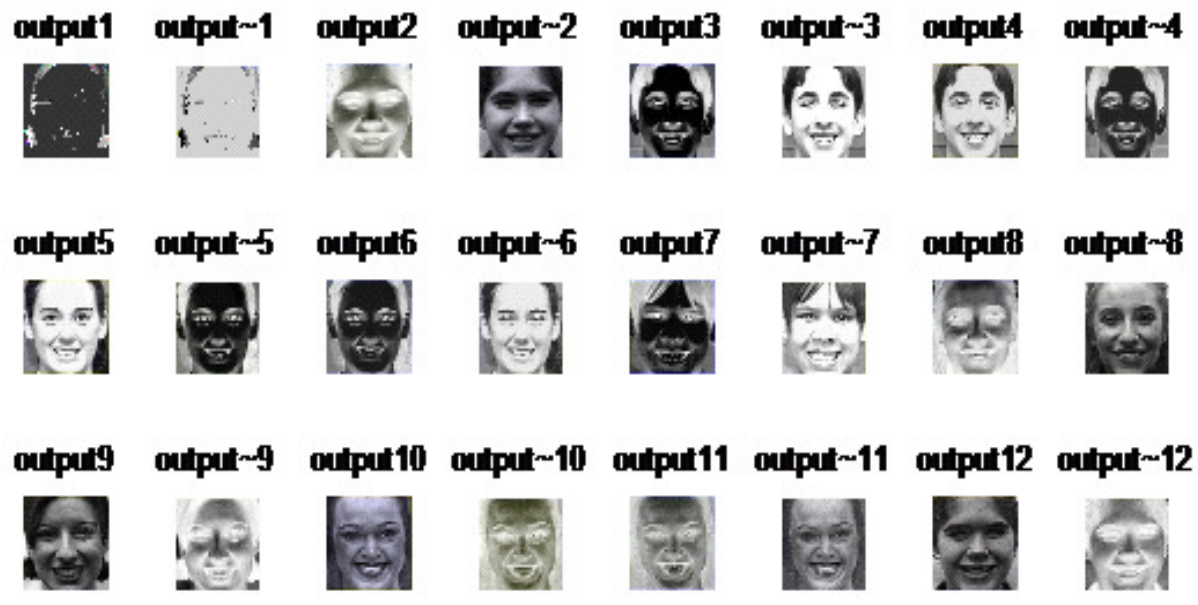

Figure 8. Independent Components

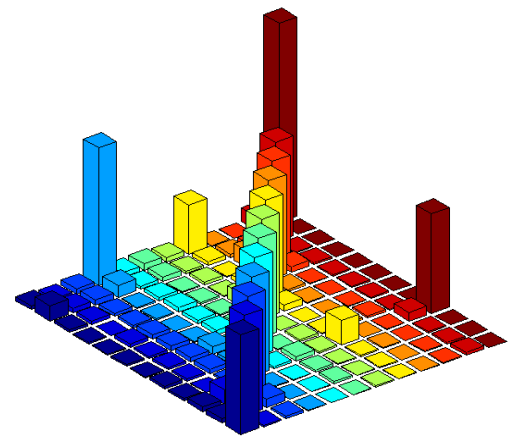

Figure 9. Global matrix 3D- Performance Index $=0.0375$ 

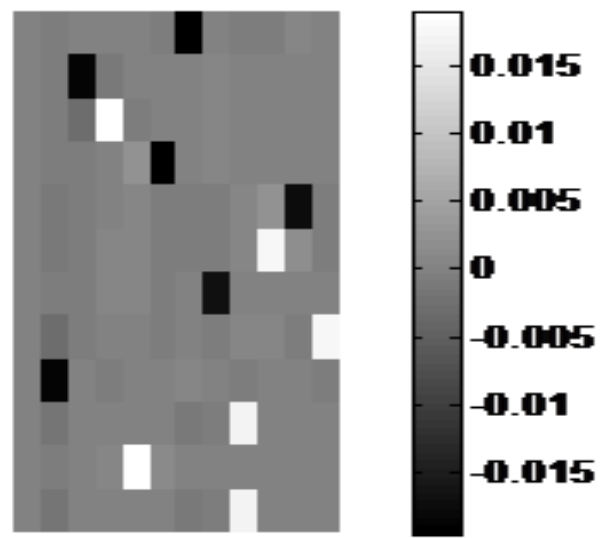

Figure 10. Estimated Matrix Inverse (W)

\subsection{FastICA with different Kernels}

We implemented FastICA with three kernels: Hyper Tangent, Gaussian and Cubic kernels. Table 1. is a comparison among first ten independent component extraction time. This table clearly shows the Gaussian kernel needs the less time compared to other kernels of FastICA. So for the next step we choose the FastICA algorithm with Gaussian kernel.

Table 1. Time comparison among different kernels of FastICA algorithm.

\begin{tabular}{|c|c|c|c|c|c|}
\hline $\begin{array}{l}\text { Algorith } \\
\mathrm{m}\end{array}$ & $\begin{array}{l}\text { Extracted } \\
\text { Component }\end{array}$ & Trial & $\begin{array}{l}\text { Iteratio } \\
n\end{array}$ & $\begin{array}{l}\text { Elapsed } \\
\text { Time }\end{array}$ & $\begin{array}{l}\text { Performance } \\
\text { Index }\end{array}$ \\
\hline $\begin{array}{l}\text { Fast ICA } \\
\text { using } \\
\text { Hyper } \\
\text { tangent } \\
(\tanh () y)\end{array}$ & $\begin{array}{l}\text { Compone } \\
\text { nt1 } \\
\text { Compone } \\
\text { nt2 } \\
\text { Compone } \\
\text { nt3 } \\
\text { Compone } \\
\text { nt4 } \\
\text { Compone } \\
\text { nt5 } \\
\begin{array}{l}\text { Compone } \\
\text { nt6 }\end{array} \\
\begin{array}{l}\text { Compone } \\
\text { nt7 }\end{array} \\
\text { Compone } \\
\text { nt8 } \\
\text { Compone } \\
\text { nt9 } \\
\text { Compone }\end{array}$ & $\begin{array}{l}1 \\
1 \\
1 \\
1 \\
1 \\
1 \\
1 \\
1 \\
1 \\
1 \\
\end{array}$ & $\begin{array}{l}11 \\
11 \\
18 \\
25 \\
13 \\
13 \\
12 \\
22 \\
16 \\
\end{array}$ & $881.57 \mathrm{~s}$ & 0.1894 \\
\hline
\end{tabular}


International Journal of Artificial Intelligence \& Applications (IJAIA), Vol. 5, No. 3, May 2014

\begin{tabular}{|c|c|c|c|c|c|}
\hline & $\mathrm{nt} 10$ & & & & \\
\hline \multirow{15}{*}{$\begin{array}{l}\text { Fast ICA } \\
\text { using } \\
\text { Gauss } \\
\left(y^{*} \text { exp }\right. \\
\left.\left(-y^{\wedge} 2 / 2\right)\right)\end{array}$} & Compone & 1 & 11 & \multirow[t]{15}{*}{$45.11 \mathrm{~s}$} & \multirow[t]{15}{*}{0.1835} \\
\hline & nt1 & 1 & 11 & & \\
\hline & Compone & 1 & 15 & & \\
\hline & nt2 & 1 & 16 & & \\
\hline & Compone & 1 & 58 & & \\
\hline & nt3 & 1 & 32 & & \\
\hline & Compone & 1 & 25 & & \\
\hline & $\mathrm{nt} 4$ & 1 & 13 & & \\
\hline & Compone & 1 & 41 & & \\
\hline & $\mathrm{nt} 5$ & 1 & 12 & & \\
\hline & $\begin{array}{l}\text { Compone } \\
\text { nt6 }\end{array}$ & & & & \\
\hline & $\begin{array}{l}\text { Compone } \\
\text { nt7 }\end{array}$ & & & & \\
\hline & $\begin{array}{l}\text { Compone } \\
\text { nt8 }\end{array}$ & & & & \\
\hline & $\begin{array}{l}\text { Compone } \\
\text { nt } 9\end{array}$ & & & & \\
\hline & Compone & & & & \\
\hline \multirow{15}{*}{$\begin{array}{l}\text { Fast ICA } \\
\text { using } \\
\text { Cubic } \\
\left(y^{\wedge} 3\right)\end{array}$} & Compone & 1 & 217 & \multirow[t]{15}{*}{$397.56 \mathrm{~s}$} & \multirow[t]{15}{*}{0.1329} \\
\hline & $\mathrm{nt} 1$ & 1 & 236 & & \\
\hline & Compone & 1 & 40 & & \\
\hline & $\mathrm{nt} 2$ & 1 & 20 & & \\
\hline & Compone & 1 & 16 & & \\
\hline & $\mathrm{nt} 3$ & 1 & 19 & & \\
\hline & Compone & 1 & 11 & & \\
\hline & nt4 & 1 & 19 & & \\
\hline & Compone & 1 & 21 & & \\
\hline & $\mathrm{nt} 5$ & \multirow[t]{6}{*}{1} & \multirow[t]{6}{*}{12} & & \\
\hline & $\begin{array}{l}\text { Compone } \\
\text { nt6 }\end{array}$ & & & & \\
\hline & $\begin{array}{l}\text { Compone } \\
\text { nt7 }\end{array}$ & & & & \\
\hline & $\begin{array}{l}\text { Compone } \\
\mathrm{nt} 8\end{array}$ & & & & \\
\hline & $\begin{array}{l}\text { Compone } \\
\text { nt } 9\end{array}$ & & & & \\
\hline & $\begin{array}{l}\text { Compone } \\
\mathrm{nt} 10\end{array}$ & & & & \\
\hline
\end{tabular}

\subsection{Influence of Different Parts}

As described before we detected facial parts like eyes, mouth and nose and applied FastIca with Gaussian kernel and calculated the weighted accuracy. Sometimes a subset of all the four parts of the face is optimal in terms of processing time and accuracy for identifying an expression. As for example from the following table (Table 2) we can see that to find the highest accuracy for fear 
both eyes and mouth are needed whereas only mouth is enough to achieve the highest accuracy of surprise expression. The other results are shown below.

Table 2: Effects of facial parts for expression recognition. LE=Left Eye, RE=Right Eye, N=Nose, $\mathrm{M}=$ Mouth, OEPA=Optimal Expression-specific Parts Accumulation

\begin{tabular}{|l|l|l|l|l|l|l|}
\hline $\begin{array}{l}\text { Facial } \\
\text { Parts }\end{array}$ & $\begin{array}{l}\text { Surpris } \\
\text { e }\end{array}$ & Anger & Sad & Happy & Fear & Disgust \\
\hline LE & $82 \%$ & $65 \%$ & $66 \%$ & $70 \%$ & $40 \%$ & $55 \%$ \\
\hline RE & $82 \%$ & $65 \%$ & $66 \%$ & $70 \%$ & $40 \%$ & $55 \%$ \\
\hline LE+RE & $82 \%$ & $65 \%$ & $66 \%$ & $70 \%$ & $40 \%$ & $55 \%$ \\
\hline $\mathrm{N}$ & $15 \%$ & $15 \%$ & $50 \%$ & $15 \%$ & $30 \%$ & $55 \%$ \\
\hline $\mathrm{M}$ & $98 \%$ & $50 \%$ & $55 \%$ & $90 \%$ & $80 \%$ & $50 \%$ \\
\hline $\begin{array}{l}\text { LE+RE+ } \\
\mathrm{N}\end{array}$ & $60 \%$ & $55 \%$ & $50 \%$ & $75 \%$ & $56 \%$ & $85 \%$ \\
\hline $\begin{array}{l}\text { LE+RE+ } \\
\mathrm{M}\end{array}$ & $98 \%$ & $80 \%$ & $70 \%$ & $100 \%$ & $96 \%$ & $80 \%$ \\
\hline N+M & $69 \%$ & $50 \%$ & $50 \%$ & $60 \%$ & $50 \%$ & $70 \%$ \\
\hline $\begin{array}{l}\text { LE+RE+ } \\
\text { N+M }\end{array}$ & $85 \%$ & $90 \%$ & $86 \%$ & $85 \%$ & $85 \%$ & $78 \%$ \\
\hline $\begin{array}{l}\text { WAPA- } \\
\text { FastICA }\end{array}$ & $92 \%$ & $88 \%$ & $82 \%$ & $88 \%$ & $90 \%$ & $82 \%$ \\
\hline $\begin{array}{l}\text { OEPA- } \\
\text { FastICA }\end{array}$ & $\begin{array}{l}98 \% \\
(\mathrm{M})\end{array}$ & $\begin{array}{l}90 \% \\
(\mathrm{LE}+\mathrm{RE}+ \\
\mathrm{N}+\mathrm{M})\end{array}$ & $\begin{array}{l}86 \% \\
(\mathrm{LE}+\mathrm{RE}+\mathrm{N} \\
+\mathrm{M})\end{array}$ & $\begin{array}{l}100 \% \\
(\mathrm{LE}+\mathrm{RE} \\
+\mathrm{M})\end{array}$ & $\begin{array}{l}96 \% \\
(\mathrm{LE}+\mathrm{RE} \\
+\mathrm{M})\end{array}$ & $\begin{array}{l}85 \% \\
(\mathrm{LE}+\mathrm{RE} \\
+\mathrm{N})\end{array}$ \\
\hline
\end{tabular}

\subsection{LS-ICA}

As described before the Locally Salient ICA (LS-ICA) has the ability to identify locally distorted parts more accurately. So we choose here LS-ICA to compare against FastICA methods even with the WAPA and OEPA based FastICA to understand which algorithm plays better role for specific applications of Facial Expression Recognition. Figure 11. shows a set of LS-ICA components after performing the LS-ICA decomposition over the images of facial expression.
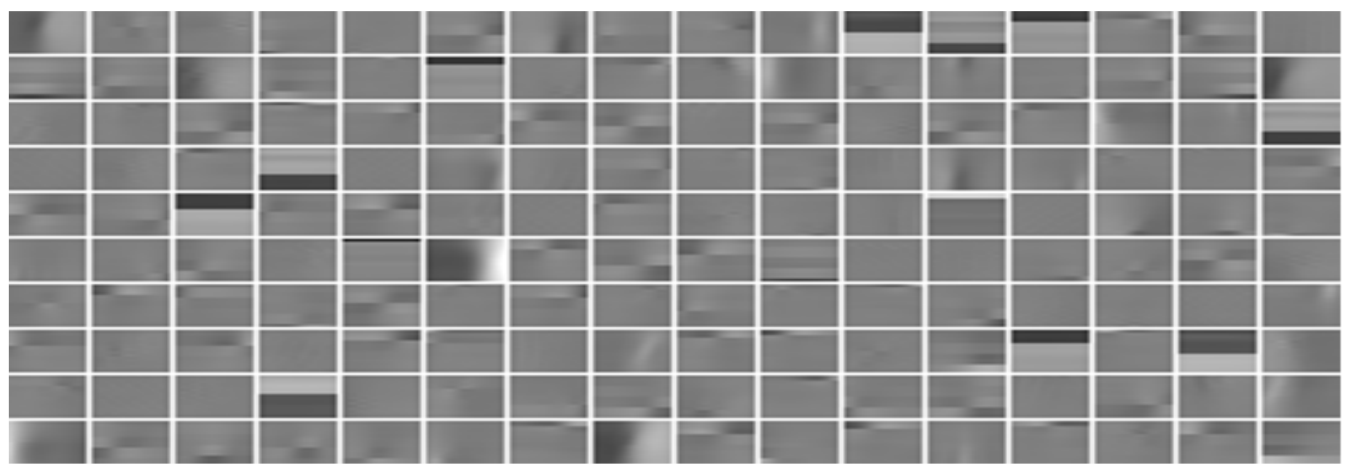

Figure 11. Locally Salient Independent components based on 8-by-8 windows and 40 dimensions. 


\section{COMPARISON AMONG ALL THE PROPOSED METHODS}

The following table (Table 3) shows the comparison among Whole face based ICA, Weighted All Parts Accunulation based FastICA, Optimal Expression Specific Parts Accumulation based FastICA, and Locally Salient ICA. For some expressions the LS-ICA outperforms the conventional FastICA and our proposed WAPA-FastICA methods. This is because LS-ICA algorithm has the strength to identify the local distortion more accurately. But the OEPA based FASTICA outperforms LS-ICA methods as we are choosing here only that facial parts which has the influence for specific facial expressions rather than choosing all the four face parts. Here we also compare these proposed methods with our proposed methods of previous published work [27]. In [27] we investigated PCA (Principal Component Analysis) on whole faces and also on different facial parts. To apply on different facial parts we propose two methods namely WAPAPCA and OEPA-PCA. In the following table and graph we will also show the comparison with these PCA methods. As we discussed in section 3, PCA works on second order derivatives and ICA on higher order derivatives and prominent features of facial expression images lie on high dimensional space. So overall recognition rate of ICA is better than PCA. Here our experiment also depicts the same evaluation.

Table 3. Comparison among proposed algorithms. (W-FastICA:Whole face ICA, WAPA:Weighted All Parts Accunulation, OEPA: Optimal Expression Specific Parts Accumulation, LS-ICA: Locally Salient ICA.)

\begin{tabular}{|l|r|r|r|r|r|r|}
\hline & Happy & \multicolumn{1}{|c|}{ Anger } & \multicolumn{1}{l|}{ Sadness } & \multicolumn{1}{l|}{ Surprise } & Fear & Disgust \\
\hline $\begin{array}{l}\text { Whole- } \\
\text { FastICA }\end{array}$ & $58 \%$ & $60 \%$ & $65 \%$ & $70 \%$ & $50 \%$ & $55 \%$ \\
\hline $\begin{array}{l}\text { WAPA- } \\
\text { FastICA }\end{array}$ & $82 \%$ & $68 \%$ & $72 \%$ & $82 \%$ & $70 \%$ & $72 \%$ \\
\hline $\begin{array}{l}\text { OEPA- } \\
\text { FastICA }\end{array}$ & $90 \%$ & $80 \%$ & $78 \%$ & $88 \%$ & $80 \%$ & $85 \%$ \\
\hline LS-ICA & $82 \%$ & $70 \%$ & $72 \%$ & $82 \%$ & $68 \%$ & $70 \%$ \\
\hline $\begin{array}{l}\text { Whole- } \\
\text { PCA }\end{array}$ & $40 \%$ & $50 \%$ & $55 \%$ & $60 \%$ & $49 \%$ & $45 \%$ \\
\hline $\begin{array}{l}\text { WAPA- } \\
\text { PCA }\end{array}$ & $78 \%$ & $62 \%$ & $66 \%$ & $70 \%$ & $60 \%$ & $60 \%$ \\
\hline $\begin{array}{l}\text { OEPA- } \\
\text { PCA }\end{array}$ & $82 \%$ & $72 \%$ & $70 \%$ & $86 \%$ & $72 \%$ & $75 \%$ \\
\hline
\end{tabular}




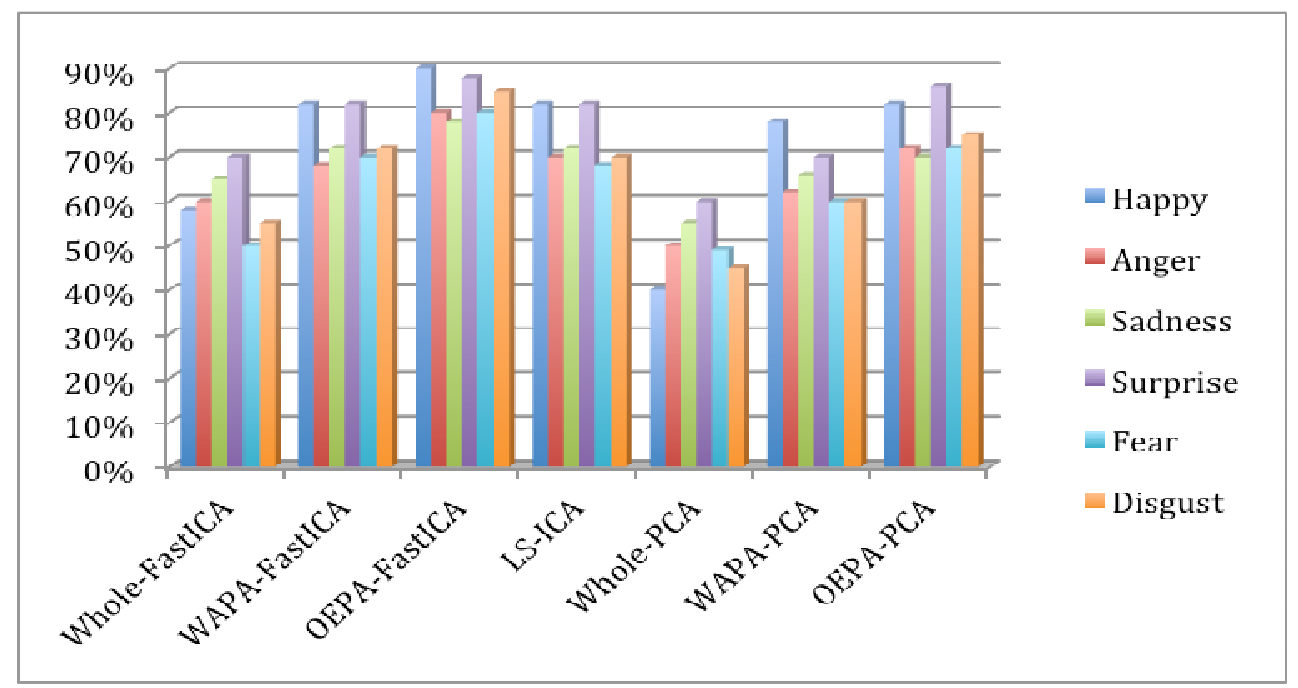

\section{CONCLUSION}

In this work, our main focus is on Independent Component Analysis (ICA). Research shows that ICA has significant success on face image analysis. Among several architectures we mainly used here FastICA and LS-ICA. We apply FastICA with Gaussian kernel on whole faces and different facial parts like, eyes, mouth and nose. When we apply ICA on whole faces, the system finds similar faces rather than similar expression when the same or similar person's face is in both train and test folder. For this reason, part based analysis, WAPA, OEPA and also LS-ICA performs better than the whole face ICA decomposition. Our experiment shows OEPA-FastICA outperforms W-FastICA, WAPA-FastICA and LS-ICA. Here we also compare these proposed methods with our proposed methods of previous published work [27]. In [27] we investigated PCA (Principal Component Analysis) on whole faces and also on different facial parts. To apply on different facial parts we propose two methods namely WAPA-PCA and OEPA-PCA. As PCA works on second order derivatives and ICA on higher order derivatives and prominent features of facial expression images lie on high dimensional space. So overall recognition rate of ICA is better than PCA and our experiment depicts the same evaluation.

\section{ACKNOWLEDGEMENTS}

This work was supported in part by the Chinese Natural Science Foundation under Grant No. 61070117, and the Beijing Natural Science Foundation under Grant No. 4122004, the Australian Research Council under ARC Thinking Systems Grant No. TS0689874 and/or Discovery Projects 0988686 and 110101473, as well as the Importation and Development of High-Caliber Talents Project of Beijing Municipal Institutions. 
International Journal of Artificial Intelligence \& Applications (IJAIA), Vol. 5, No. 3, May 2014

\section{REFERENCES}

[1] A. Hyvarinen, (1999) "Survey of Independent Component Analysis", Neural Computing Surveys, 2:94-128.

[2] A.Hyvarinen \& E.Oja, (2000) "Independent component analysis: Algorithms and application", Neural Networks, 13(4-5):411- 430.

[3] A. Hyvarinen \& E. Oja, (1997) "A fast fixed-point algorithm for independent component analysis", Neural Computation, 9(7): 1483-1492.

[4] A. Hyvarinen, R.Cristescu, \& E.Oja, (1999) "A fast algorithm for estimating overcomplete ICA bases for image windows", Proc.Int.Joint Conf. on Neural Networks, Pages 894-899, Washington, D.C.

[5] T. Sergios, Pattern Recognition Book.

[6] Barlow H.B., (1989) "Unsupervised Learning”, Neural Computation, Vol. 1,pp. 295-311.

[7] S.B. Marian, J.R. Movellan \& T. J. Sejnowski, (2002) "Face recognition by ICA", IEEE Transactions on Neural Networks.

[8] S. B. Marian, R. M. Javier, \& J. S. Terrence, (2002) "Face Recognition by Independent Component Analysis", IEEE transaction Neural Networks, 13(6), 1450-1464.

[9] H. B. Barlow, (1989) "Unsupervised learning," Neural Computing, vol. 1, pp. 295-311.

[10] J. J. Atick \& A. N. Redlich, (1992) "What does the retina know about natural scenes?," Neural Comput., vol. 4, pp. 196-210.

[11] G. Donato, M. S. Bartlett, J. C. Hagar, P. Ekman, \& T. J. Sejnowski, (1999) "Classifying Facial Actions," IEEE Transaction on Pattern Analysis and Machine Intelligence, vol. 21, no. 10, pp. 974989.

[12] P. Ekman \& W. V. Priesen, (1978) "Facial Action Coding System: A technique for the Measurement of Facial Movement," Consulting Psychologists Press, Palo Alto, CA. Contributed Paper Manuscript received September 28, 20090098 3063/09/\$20.00 @ 2009 IEEE

[13] I. Buciu, C. Kotropoulos, \& I. Pitas, (2003) "ICA and Gabor Representation for Facial Expression Recognition," in Proceedings of the IEEE, pp. 855-858.

[14] F. Chen \& K. Kotani, (2008) "Facial Expression Recognition by Supervised Independent Component Analysis Using MAP Estimation,” IEICE Transactions on Information and Systems, vol. E91-D, no. 2, pp. 341-350.

[15] A. Hyvarinen, J. Karhunen, \& E. Oja, (2001) "Independent Component Analysis," John Wiley \& Sons.

[16] Y. Karklin \& M.S Lewicki, (2003) "Learning higher-order structures in natural images," Network: Computation in Neural Systems, vol. 14, pp. 483-499.

[17] M. S. Bartlett, G. Donato, J. R. Movellan, J. C. Hager, P. Ekman, \& T. J. Sejnowski, (1999) "Face Image Analysis for Expression Measurement and Detection of Deceit," in Proceedings of the Sixth Joint Symposium on Neural Computation, pp. 8-15.

[18] C. Chao-Fa \& F. Y. Shin, (2006) "Recognizing Facial Action Units Using Independent Component Analysis and Support Vector Machine," Pattern Recognition, vol. 39, pp. 1795-1798.

[19] A. J. Calder, A. W. Young, \& J. Keane, (2000) "Configural information in facial expression perception," Journal of Experimental Psychology: Human Perception and Performance, vol. 26, no. 2, pp. 527-551.

[20] M. J. Lyons, S. Akamatsu, M. Kamachi, \& J. Gyoba, (1998) "Coding facial expressions with Gabor wavelets," in Proceedings of the Third IEEE International Conference on Automatic Face and Gesture Recognition, pp.200-205.

[21] M. S. Bartlett, J. R. Movellan, \& T. J. Sejnowski, (2002) "Face Recognition by Independent Component Analysis," IEEE Transaction on Neural Networks, vol. 13, no. 6, pp. 1450-1464.

[22] C. Liu, (2004) "Enhanced independent component analysis and its application to content based face image retrieval," IEEE Transactions on Systems, Man, and Cybernetics-Part B: Cybernetics, vol. 34, no. 2, pp. 1117- 1127.

[23] P. J. Phillips, H. Wechsler, J. Huang, \& P. Rauss, (1998) "The FERET database and evaluation procedure for face-recognition algorithms," Image and Vision Computing, vol. 16, pp. 295-306. 
International Journal of Artificial Intelligence \& Applications (IJAIA), Vol. 5, No. 3, May 2014

[24] A. B. Humayra, P. M. W. David, L. Richard \& L. W. Trent, (2011) "Comparison of Region Based and Weighted Principal Component Analysis and Locally Salient ICA in Terms of Facial Expression Recognition", 81-89. International Conference on Software Engineering, Artificial Intelligence, Networking and Parallel/Distributed Computing (SNPD), Springer, Sydney, Australia.

[25] A. B. Humayra and P. M. W. David, (2013) "Facial expression recognition based on Weighted All Parts Accumulation and Optimal Expression-specific Parts Accumulation", The InternationalConference on Digital Image Computing: Techniques and Applications (DICTA) Hobart, Tasmania, Australia, page 229-235.

\section{Authors Short Biography}

1.Humayra Binte Ali is currently pursuing $\mathrm{PhD}$ in CSEM School of Flinders University, South Australia.She graduated form the dept of Computer science and Engineering, Dhaka University, Bangladesh, in 2007 with a first class honours. She was involved in research project of MAGICIAN, Multiple Autonomous Groundrobotic International Challenge by Intelligent Adelaide Navigators(2009-2010), in Flinders University. She also worked as a research assistant in Deakin University, Melbourne with the project of "Early Heart Rate Detection with Machine

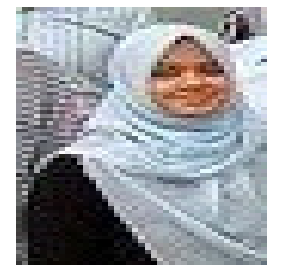
Learning". Her research interests are in pattern recognition, machine learning, computer vision, image analysis, cognitive neuroscience and facial

expression recognition.

\section{Professor David M W Powers}

Beijing Municipal Lab for Multimedia \& Intelligent Software

Beijing University of Technology

Beijing, China

KIT Centre, School of Comp.Sci., Eng. \& Maths

Flinders University

Adelaide, South Australia

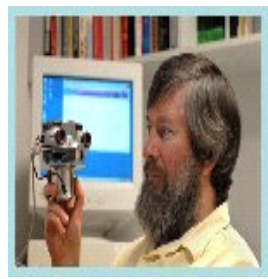

